\title{
Immunomodulation Mediated by Anti-angiogenic Therapy Improves CD8 T Cell Immunity Against Experimental Glioma
}

\author{
Courtney S. Malo ${ }^{1,2}$, Roman H. Khadka ${ }^{1,2}$, Katayoun Ayasoufi ${ }^{1}$, Fang Jin ${ }^{1}$, \\ Jackson E. AbouChehade ${ }^{3}$, Michael J. Hansen ${ }^{1}$, Raymond lezzi ${ }^{3}$, Kevin D. Pavelko ${ }^{1}$ and \\ Aaron J. Johnson ${ }^{1,4,5 *}$ \\ 'Department of Immunology, Mayo Clinic, Rochester, MN, United States, ${ }^{2}$ Mayo Clinic Graduate School of Biomedical \\ Sciences, Mayo Clinic, Rochester, MN, United States, ${ }^{3}$ Department of Ophthalmology, Mayo Clinic, Rochester, MN, \\ United States, ${ }^{4}$ Department of Neurology, Mayo Clinic, Rochester, MN, United States, ${ }^{5}$ Department of Molecular Medicine, \\ Mayo Clinic, Rochester, MN, United States
}

OPEN ACCESS

Edited by:

Shiv K. Gupta

Mayo Clinic, United States

Reviewed by:

Thomas Daubon,

Institut National de la Santé et de la

Recherche Médicale (INSERM),

France

Sujuan Guo,

Dana-Farber Cancer Institute,

United States

*Correspondence:

Aaron J. Johnson

Johnson.aaron2@mayo.edu

Specialty section:

This article was submitted to

Cancer Molecular Targets and

Therapeutics,

a section of the journa

Frontiers in Oncology

Received: 15 June 2018

Accepted: 26 July 2018

Published: 20 August 2018

Citation:

Malo CS, Khadka RH, Ayasoufi K, Jin F, AbouChehade JE, Hansen MJ, lezzi R, Pavelko KD and Johnson AJ (2018) Immunomodulation Mediated by Anti-angiogenic Therapy Improves

CD8 T Cell Immunity Against

Experimental Glioma.

Front. Oncol. 8:320.

doi: 10.3389/fonc.2018.00320
Glioblastoma (GBM) is a lethal cancer of the central nervous system with a median survival rate of 15 months with treatment. Thus, there is a critical need to develop novel therapies for GBM. Immunotherapy is emerging as a promising therapeutic strategy. However, current therapies for GBM, in particular anti-angiogenic therapies that block vascular endothelial growth factor (VEGF), may have undefined consequences on the efficacy of immunotherapy. While this treatment is primarily prescribed to reduce tumor vascularization, multiple immune cell types also express VEGF receptors, including the most potent antigen-presenting cell, the dendritic cell (DC). Therefore, we assessed the role of anti-VEGF therapy in modifying DC function. We found that VEGF blockade results in a more mature DC phenotype in the brain, as demonstrated by an increase in the expression of the co-stimulatory molecules B7-1, B7-2, and MHC II. Furthermore, we observed reduced levels of the exhaustion markers PD-1 and Tim-3 on brain-infiltrating CD8 T cells, indicating improved functionality. Thus, anti-angiogenic therapy has the potential to be used in conjunction with and enhance immunotherapy for GBM.

Keywords: glioblastoma, anti-angiogenic therapy, immunotherapy, vaccine, combination therapy

\section{INTRODUCTION}

Glioblastoma (GBM) is a lethal cancer of the central nervous system (CNS). Patients diagnosed with GBM have a median expected survival of about 15 months following diagnosis with treatment $(1,2)$. As it currently stands, there is no cure for GBM, and even with surgical resection of the tumor, a patient will universally recur and succumb to disease. Therefore, there is a clear need for the development of new therapies for GBM treatment.

One such therapeutic strategy that has been rising in popularity are immunotherapies, which aim to target the immune system to respond to the tumor. Immunotherapies provide a facet of precision not possible with surgical techniques, which are unable to target the invasive edges of the tumor, or chemotherapies, which nonspecifically target all dividing cells (3). As a result, numerous research groups are testing a variety of immunotherapy strategies against GBM tumors, both in pre-clinical models and in clinical trials (2). In particular, strategies to activate tumor 
antigen-specific CD8 T cells, which will then kill tumor cells using cytotoxic granules, have been promising (4). While these therapies have demonstrated some success, there are still no curative strategies for GBM. This is primarily due to the immune suppressive nature of the tumor microenvironment, and the global immune dysregulation patients present with despite immunotherapy treatments.

To simultaneously bypass the immune suppressive tumor environment and stimulate anti-tumor immune responses, concomitant therapies have become highly prevalent. These treatment regimens often combine a therapy that is currently in use with a novel immunotherapy, including vaccination (2). Importantly, the synergy between many of these combination treatments has not been defined. For example, combining antiangiogenic therapies, often used in patients with recurrent GBM following surgical resection, with immunotherapies, improves survival in pre-clinical models (5). However, the extent to which anti-angiogenic therapy blocking vascular endothelial growth factor (VEGF) impacts the immune response to GBM directly, is unclear. Studies in other tumor models and in in vitro assays have suggested a regulatory role of VEGF on the immune system $(6,7)$. These studies in particular demonstrate a role for VEGF on retention of dendritic cells, a potent antigen presenting cell (APC), in state of reduced activation. This would in turn reduce $T$ cell activation and subsequently negate the impact $T$ cell-based immunotherapy strategies, including tumor antigenspecific vaccination.

We hypothesized that blockade of VEGF using the clinically available anti-angiogenic therapy, VEGF-Trap (Eylea/Aflibercept), we would improve dendritic cell maturation and in turn improve antitumor $\mathrm{T}$ cell responses in a murine model of GBM, the GL261-quad cassette syngeneic glioma. Our group has previously demonstrated that treatment with VEGF-Trap, which is a VEGF receptor (VEGFR) fusion protein conjugated to a human IgG FC region, results in similar outcomes as GBM patients treated with bevacizumab antiangiogenic therapies as measured by T1- and T2-weighted magnetic resonance imaging (MRI) and histology (5). Likewise, VEGF-trap treatment improves survival in GL261-quad cassette bearing animals (5). Importantly, VEGF-Trap is used in place of bevacizumab due to improved cross-reactivity with murine VEGF (8).

To address this hypothesis, we first assessed the expression of VEGFRs on the surface of dendritic cells, which we contend are the most potent APC to generate CD8 T cell responses in the CNS (9). We also treated GL261-quad cassette bearing animals with VEGF-Trap weekly and assessed the quality of dendritic cell activation in the tumor draining lymph nodes (TLDNs) 14 days post treatment. We also evaluated the proportion of tumor antigen-specific CD8 T cells in the CNS of these animals.

\section{MATERIALS AND METHODS}

\section{Acute Viral Infection and Vaccination}

Six- to eight-week-old C57BL/6 mice were infected intracranially (i.c.) with Theiler's murine encephalomyelitis virus (TMEV) as previously described (9-11). Animals were anesthetized with $1-2 \%$ isoflurane, then received a single dose of $2 \times$ $10^{5}$ plaque forming units (PFU) of TMEV in the right hemisphere of the brain. VEGFR expression was measured in the draining lymph nodes and brain 5 and 7 days following infection.

\section{GL261 Cell Culture and Implantation}

The GL261-quad cassette cell line has been transgenically modified to express four model antigens: OVA $257-264$,

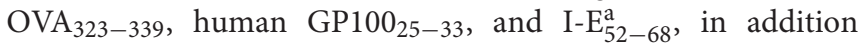
to a luciferase transgene to assess tumor burden. $6 \times 10^{4}$ GL261quad cassette cells were implanted by stereotactic injection as previously described $(5,10)$. Six- to eight-week-old female C57BL/6 animals were anesthetized with $20 \mathrm{mg} / \mathrm{kg}$ ketamine and $5 \mathrm{mg} / \mathrm{kg}$ xylazine to minimize discomfort during the procedure. Cells were injected at a concentration of $6 \times 10^{4}$ GL261 cells per $1 \mu \mathrm{L}$ phosphate buffered saline (PBS). Injection rate was 0.2 $\mu \mathrm{L}$ per minute. The site of injection was $1 \mathrm{~mm}$ lateral, $2 \mathrm{~mm}$ anterior of the bregma with a depth of $3 \mathrm{~mm}$ from the surface. All animal experiments were approved by and performed in accordance with the Mayo Clinic Institutional Animal Care and Use Committee.

\section{Bioluminescence Imaging}

GL261-quad cassette-bearing animals were assessed for tumor burden using bioluminescence imaging as previously described $(5,10)$. Animals were intraperitoneally injected with $150 \mathrm{mg} / \mathrm{kg}$ D-luciferin sodium salt in PBS (Gold Biotechnology, Olivette, $\mathrm{MO})$. Animals were anesthetized with $1-2 \%$ isoflurane before and throughout imaging. Animals were scanned using an IVIS Spectrum system (Xenogen Corp., Amameda, CA, USA) running Living Image software. Bioluminescence intensity (photons/s) was recorded in a circular region of interest surrounding the head. Animals with average bioluminescence intensity above $10^{5}$ photons/s were considered tumor bearing and treated with VEGF-Trap or PBS. All animal work was completed in accordance to the Mayo Clinic Institutional Animal Care and Use Committee guidelines.

\section{Anti-angiogenic Therapy Treatment}

VEGF-Trap/Aflibercept (Regeneron Pharmaceuticals, Rensselaer, NY, USA) was administered at a dose of $12.5 \mathrm{mg} / \mathrm{kg}$ in PBS in a total volume of $100 \mu \mathrm{L}$ intravenously (i.v.) by injection into the tail vein 2 weeks post-tumor injection. Treatment was continued weekly until animals were euthanized for flow cytometry analysis. Control mice received $100 \mu \mathrm{L}$ PBS i.v., at the same time points.

\section{Flow Cytometry}

Lymph nodes and spleens were harvested in RPMI and pressed through a $70 \mu \mathrm{m}$ filter to achieve a single cell suspension for compensation control samples. Brains were harvested and manually homogenized using a dounce homogenizer as previously described (12). Brain samples were filtered through a $70 \mu \mathrm{m}$ filter to achieve a single cell suspension into a $50 \%$ percoll solution. Samples were centrifuged at 7,840 g. The myelin 
debris layer formed at the top of the gradient was aspirated. All samples were washed twice and plated in a 96-well v bottom plate. Peptide:MHC tetramers were constructed by our lab and samples were stained at a 1:100 dilution of tetramer for $30 \mathrm{~min}$ at room temperature in the dark. Following a wash, antibodies against CD45, CD11c, CD11b, I-A b, CD80, CD86, VEGFR2, Nrp-1, CD4, CD8 $\alpha$, PD-1, and Tim-3 were used for staining at a 1:100 dilution for $30 \mathrm{~min}$ on ice in the dark (BD Biosciences, San Jose, CA; Tonbo Biosciences, San Diego, CA) in addition to Ghost Red 780 Viability Dye used at a 1:1000 dilution (Tonbo Biosciences, San Diego, CA). Samples were fixed with 2\% paraformaldehyde. Samples were subsequently run on a BD LSRII flow cytometer equipped with FACSDiva software (BD Biosciences, San Jose, CA). Samples were digitally compensated using single-stained controls and analyzed by FlowJo v10 software (FlowJo LLC, Ashland, OR).

\section{Statistical Analysis}

All data are presented as mean \pm standard error of the mean (SEM). Significance was determined using a Mann-Whitney Rank Sum Test. GraphPad Prism 7.0 (La Jolla, CA) were used for all statistical analysis.

\section{Data Availability}

All data generated during this study are available from the corresponding author on reasonable request.

\section{RESULTS}

\section{Dendritic Cells Express VEGFRs in the Inflamed CNS}

To address the impact of VEGF signaling on the immune system, we first sought to identify the cells through which VEGF would signal. To address this question, we used infection with Theiler's Murine Encephalomyelitis Virus (TMEV) as a model of CNS inflammation. Intracranial infection with TMEV results in extensive immune cell expansion in the deep cervical lymph nodes and subsequent immune cell infiltration into the CNS (13). We therefore assessed VEGFR expression on $\mathrm{CD}_{11 \mathrm{c}^{+}}$ dendritic cells, compared to $\mathrm{CD}_{11 \mathrm{c}^{-}}$immune cells, 5 and 7 days post infection. Expression of VEGFR2, considered the primary signaling receptor for VEGF, and Neuropilin-1, known as a co-receptor for VEGF signaling. We found that CD11c ${ }^{+}$ dendritic cells express low but detectable levels of VEGFR2 in the deep cervical lymph nodes 5 days post infection, and express higher levels of VEGFR2 in the brain 5 days post infection (Figures 1A,B). By 7 days post infection, CD11c ${ }^{+}$ cells in the brain express high levels of both VEGFR2 and neuropilin-1, suggesting that dendritic cells in the CNS are capable of signaling through VEGF receptors (Figures 1A,B). This also suggests that neuropilin-1 expression is induced following inflammation. Notably, we do not see upregulation of VEGFR2 or neuropilin-1 expression on $\mathrm{CD}_{11 c^{-}}$immune cells.

We next evaluated VEGFR expression on antigen presenting cells (APCs) in the brain in unvaccinated C57BL/6 mice harboring established GL261 gliomas. We primarily focused on dendritic cells owing to its dominant role in mounting anti-glioma response (9). We determined that dendritic cells isolated from the brain of these animals expressed VEGFR2 at higher levels than $\mathrm{CD} 45+, \mathrm{CD} 11 \mathrm{c}^{-}$blood derived cell types (Figure 1C). Furthermore, the proportion and absolute counts of isolated dendritic cells that express VEGFR2 is significantly higher in glioma bearing mice (Figures 1D,E). We further assessed the expression levels of VEGFRs on other brain-infiltrating and resident immune cells as well. We found the VEGFR2 levels on CD45 ${ }^{\text {int }} \mathrm{CD} 11 \mathrm{~b}^{+}$microglial cells remained unchanged in comparison to non-tumor bearing littermates (data not shown). Similarly, the expression level on other $\mathrm{CD} 45+$, CD11c- immune cell types was unremarkable (Figures 1C-E).

\section{Dendritic Cells Are More Activated, and CD8 T Cells Are Less Exhausted, Following Anti-angiogenic Therapy}

After demonstrating that dendritic cells express VEGFRs, we next sought to determine the impact of this expression on anti-glioma immune responses. To accomplish this, we implanted GL261quad cassette gliomas in C57BL/6 mice. Two weeks following tumor implantation, we imaged animals using bioluminescence imaging to remove animals from the study that did not bear tumors. We treated only tumor-bearing animals with VEGFTrap intravenously. A second cohort of animals was treated with PBS as a control. Two weeks following treatment, or 4 weeks post-tumor implantation, brains were harvested and processed for flow cytometric analysis.

Dendritic cells isolated from the brains of tumor bearing animals were assessed for expression of known activation markers, including CD80 (B7-1), CD86 (B7-2), and I-A ${ }^{\mathrm{b}}$ major histocompatibility complex (MHC) class II. We found that following VEGF-Trap treatment, a higher proportion of dendritic cells expressed each of these markers, as compared with PBS treatment (Figures 2A-D). These markers are required for $\mathrm{T}$ cell activation, and increase in each of these markers suggests that VEGF-Trap treatment results in dendritic cells that are better capable of stimulating an anti-tumor immune response.

We next assessed the impact VEGF-Trap treatment had on brain infiltrating, tumor antigen-specific CD8 T cells. To accomplish this, we measured expression of the exhaustion markers PD-1 and Tim-3 (14). We assessed proportion of cells expressing these markers on both total CD8 T cells and on $\mathrm{K}^{\mathrm{b}}$ : OVA-specific CD8 T cells, as the GL261-quad cassette cell line expresses OVA peptide (SIINFEKL) as a model tumor antigen (10). We determined that fewer CD8 T cells infiltrating the brain following VEGF-Trap treatment had high expression of PD-1 and Tim-3 (Figures 2E-H). Therefore, a reduced proportion of $\mathrm{CD} 8 \mathrm{~T}$ cells are exhausted as a result of VEGF-Trap treatment. Furthermore, tumor antigen-specific CD8 $\mathrm{T}$ cells, defined as being $\mathrm{Kb}$ :OVA Tetramer ${ }^{+}$, are also less exhausted than tumor antigen-specific CD8 cells isolated from PBS treated animals (Figures 2I,J). These findings suggest that VEGF-Trap treatment results in a tumor-specific CD8 $\mathrm{T}$ cell 

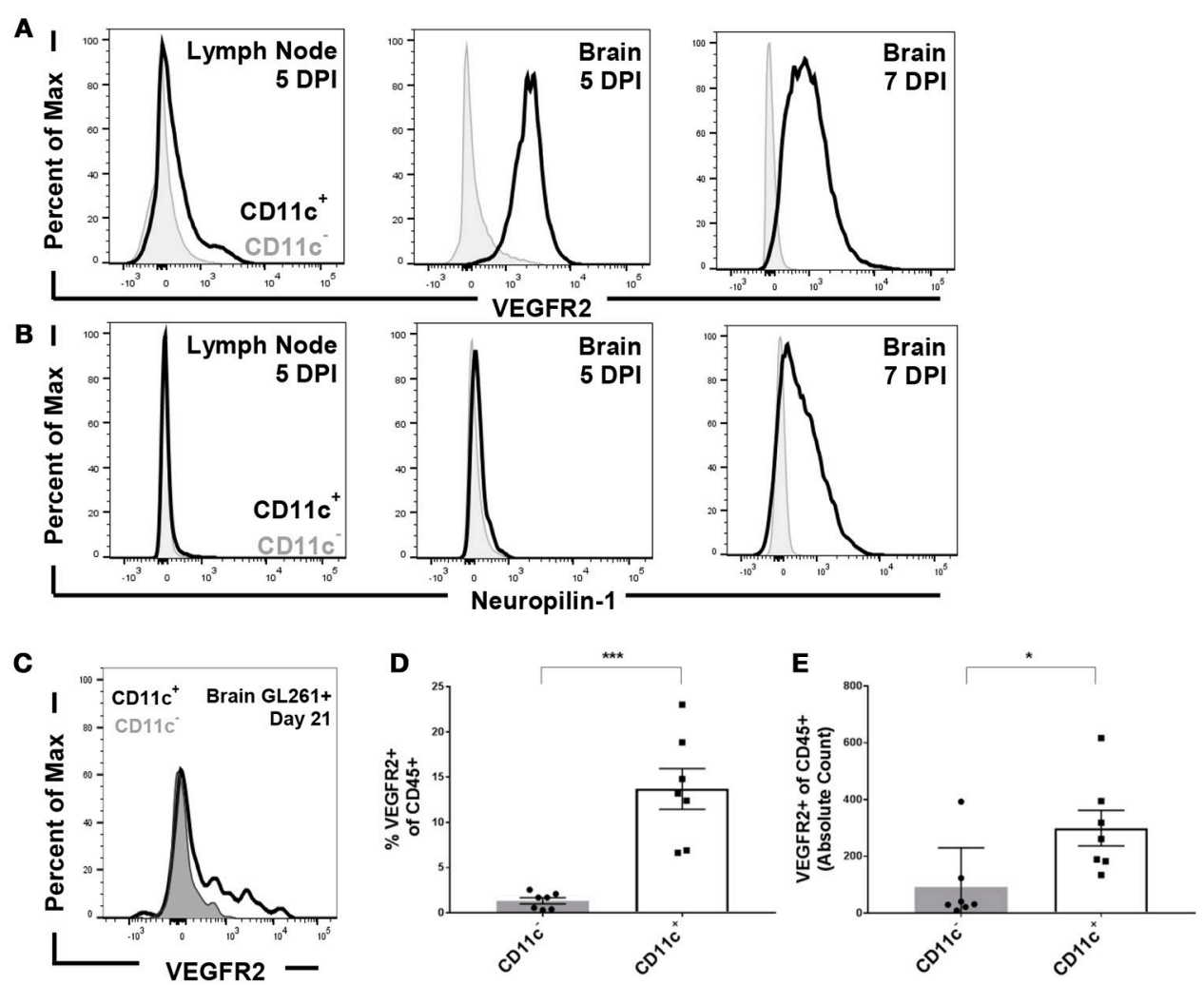

FIGURE 1 | Dendritic cells express VEGFR2 and neuropilin-1 in the brain during picornavirus infection and 21 day established GL261 glioma. Dendritic cells were isolated from the cervical lymph node and brain five and seven DPI. Dendritic cells were gated as CD45 hi and CD11C ${ }^{+}$. (A) Dendritic cells in the lymph node and brain express VEGFR2, with a majority of dendritic cells expressing VEGFR2 seven DPI in the brain. (B) Brain-infiltrating dendritic cells express neuropilin-1, a co-receptor for VEGF, in the brain seven DPI with TMEV. (C) Representative flow plot showing expression of VEGFR2 in both CD11 $\mathrm{C}^{+}$and $\mathrm{CD}_{11 \mathrm{c}^{-}}$cells isolated from the brain of unvaccinated animal bearing GL261 glioma. (D,E) In untreated mice with 21 day established GL261 gliomas, we observed CD11C+ dendritic cells in the brain express higher levels of VEGFR2 $(N=7)$. Data presented as mean with error bars representing standard error of the mean $(\mathrm{SEM}) .{ }^{*} p \leq 0.05$ and ${ }^{* * *} p \leq 0.001$ by Mann-Whitney U-Test.

response that is more capable of carrying out their cytotoxic effector function.

\section{DISCUSSION}

Here we demonstrate that VEGF-Trap treatment, as one example of anti-angiogenic therapy, results in a treatment response beyond vasculature normalization. In addition to the previously demonstrated effects observed by this treatment in the GL261 glioma model, we observe a significant change in dendritic cell maturation status and in CD8 T cell exhaustion. These findings are of great importance as immunotherapies are developed for CNS cancers.

Dendritic cell maturation is key for effective antigen presentation of tumor antigens. This is true for both generation of an endogenous immune response as well as in the context of vaccination. We demonstrate that dendritic cells isolated from the lymph nodes of VEGF-Trap treated animals exhibit enhanced expression of costimulatory molecules such as CD80, CD86, and MHC class II. Therefore, dendritic cells from VEGF-Trap treated animals have the capacity to be better antigen presenting cells. Likewise, CD8 T cells isolated from VEGF-Trap treated animals have a demonstrable decrease in exhaustion markers. CD8 $\mathrm{T}$ cell exhaustion has been shown to be mediated by the immune suppressive tumor microenvironment, and VEGF is likely one way this is accomplished (14). Much like through the use of checkpoint blockade therapy, if the signals that result in CD8 T cell exhaustion can be prevented through VEGF blockade, the CD8 T cells that infiltrate the tumor will be better able to kill tumor cells. Furthermore, these findings are not limited to just cancers of the CNS. Anti-angiogenic therapy is used in colorectal cancer and breast cancer treatment $(15,16)$. Likewise, immunotherapies are being tested in both of these types of cancer $(17,18)$. Therefore, our findings may be extrapolated to other combination strategies involving an immune therapy and anti-angiogenic therapy.

Here we show that anti-angiogenic therapy is not only a useful strategy to improve quality of life for patients diagnosed with GBM, but it may be a tractable approach to enhance immunotherapies. This study also builds upon our previous publication in which it was determined that combination 
A
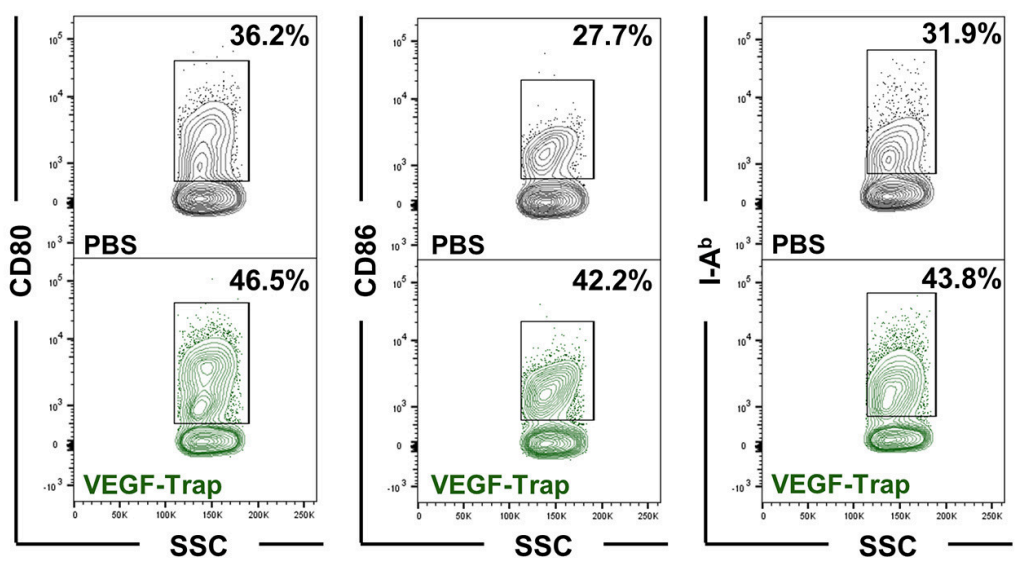

B

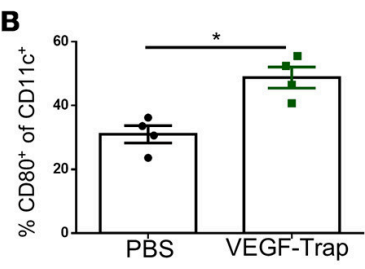

C

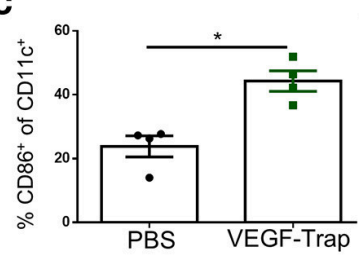

D

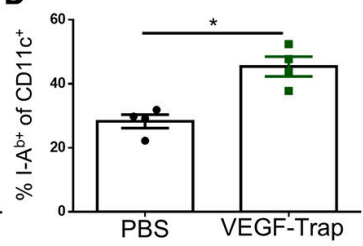

E

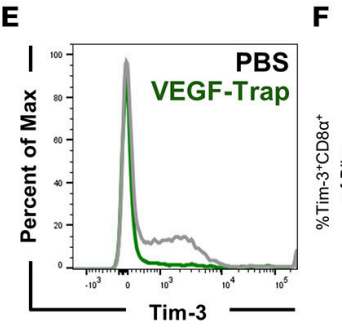

G

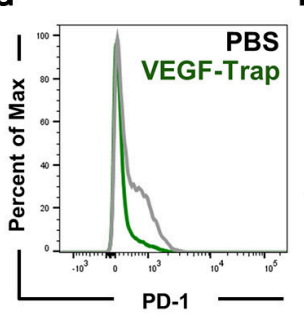

H

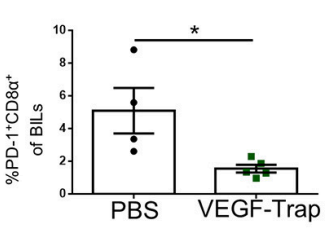

I

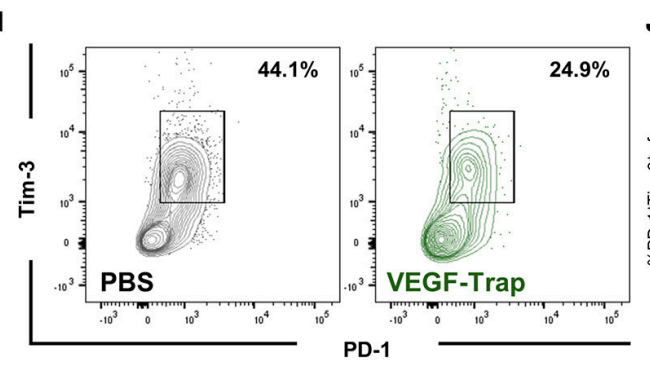

$J$

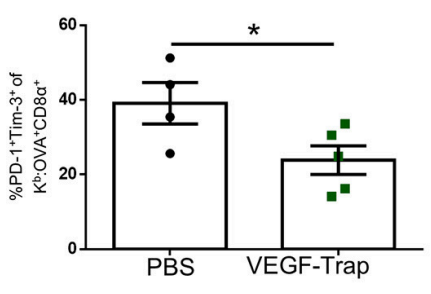

FIGURE 2 | CD8 T cells and dendritic cells isolated from the brain of VEGF-Trap treated GL261-quad cassette bearing mice express a more functional phenotype. GL261-quad cassette bearing animals were treated with PBS $(N=4)$ or VEGF-Trap $(N=5) 2$ weeks post-tumor implantation. Animals were sacrificed 30 days after tumor implantation and brain infiltrating leukocytes (BILs) were assessed by flow cytometry. (A) Representative images of CD11 $\mathrm{C}^{+}$cells isolated from the brain assessing expression of costimulatory markers. VEGF-Trap treatment results in increased expression of CD80 (B), CD86 (C), and I-A ${ }^{b}$ MHC Class II (D). Representative flow plots (E) and quantification (F) show reduction in the proportion of Tim-3+ CD8 T cells in the CNS of GL261-quad cassette bearing animals. Representative flow plots $(\mathbf{G})$ and quantification $(\mathbf{H})$ show a reduction in the proportion of CD8 T cells expressing PD-1 in the brain. A reduction in PD- $1^{+}$Tim- $3^{+}$ double positive CD8T cells was also observed (I,J). Error bars represent mean \pm SEM. ${ }^{*} p<0.05$. Side Scatter (SSC) was included as a measure of granularity.

therapy of picornavirus vaccination plus antiangiogenic treatment extended that lifespan of mice harboring GL261 gliomas (5). Therefore, we contend that we have identified another candidate for the family of checkpoint blockade treatments. VEGF blockade should be considered in preclinical models of immunotherapies to dually normalize the vasculature and enhance tumor antigen-specific CD8 T cell responses. 


\section{AUTHOR CONTRIBUTIONS}

CM, RK, KA, RI, KP, and AJ: Conceptualization; CM, RK, KA, and AJ: Formal analysis; AJ: Funding acquisition; $\mathrm{CM}, \mathrm{RK}, \mathrm{KA}, \mathrm{FJ}$, and $\mathrm{AJ}$ : Investigation; $\mathrm{CM}, \mathrm{RK}$, and AJ: Methodology; AJ: Project administration; JA, RI, and KP: Resources; AJ: Supervision; CM: Visualization; writingoriginal draft; CM, RK, KA, and AJ: Writing-reviewing and editing.

\section{REFERENCES}

1. Alifieris C, Trafalis DT. Glioblastoma multiforme: pathogenesis and treatment. Pharmacol. Ther. (2015) 152:63-82. doi: 10.1016/j.pharmthera.2015.05.005

2. Preusser M, Lim M, Hafler DA, Reardon DA, Sampson JH. Prospects of immune checkpoint modulators in the treatment of glioblastoma. Nat Rev Neurol. (2015) 11:504-14. doi: 10.1038/nrneurol.2015.139

3. Omuro A, DeAngelis LM. Glioblastoma and other malignant gliomas: a clinical review. JAMA (2013) 310:1842-50. doi: 10.1001/jama.2013.280319

4. Xu LW, Chow KK, Lim M, Li G. Current vaccine trials in glioblastoma: a review. J Immunol Res. (2014) 2014:796856. doi: 10.1155/2014/796856

5. Renner DN, Malo CS, Jin F, Parney IF, Pavelko KD, Johnson AJ. Improved treatment efficacy of antiangiogenic therapy when combined with picornavirus vaccination in the GL261 glioma model. Neurotherapeutics (2016) 13:226-36. doi: 10.1007/s13311-015-0407-1

6. Heine A, Held SA, Bringmann A, Holderried TA, Brossart P. Immunomodulatory effects of anti-angiogenic drugs. Leukemia (2011) 25:899-905. doi: 10.1038/leu.2011.24

7. Zdravkovic ND, Jovanovic IP, Radosavljevic GD, Arsenijevic AN, Zdravkovic ND, Mitrovic S, et al. Potential dual immunomodulatory role of VEGF in ulcerative colitis and colorectal carcinoma. Int J Med Sci. (2014) 11:936-47. doi: 10.7150/ijms.8277

8. Irani Y, Scotney P, Nash A, Williams KA. Species cross-reactivity of antibodies used to treat ophthalmic conditions. Invest Ophthalmol Vis Sci. (2016) 57:586-91. doi: 10.1167/iovs.15-18239

9. Malo CS, Huggins MA, Goddery EN, Tolcher HMA, Renner DN, Jin F, et al. Non-equivalent antigen presenting capabilities of dendritic cells and macrophages in generating brain-infiltrating $\mathrm{CD}^{+} \mathrm{T}$ cell responses. Nat Commun. (2018) 9:633. doi: 10.1038/s41467-018-03037-x

10. Renner DN, Jin F, Litterman AJ, Balgeman AJ, Hanson LM, Gamez JD, et al. Effective treatment of established GL261 murine gliomas through picornavirus vaccination-enhanced tumor antigen-specific CD8 ${ }^{+} \mathrm{T}$ cell responses. PLoS ONE (2015) 10:e0125565. doi: 10.1371/journal.pone.0125565

11. Malo CS, Renner DN, Huseby Kelcher AM, Jin F, Hansen, MJ, Pavelko KD, et al. (2016). The effect of vector silencing during picornavirus vaccination against experimental melanoma and glioma. PLoS ONE 11:e0162064. doi: 10.1371/journal.pone.0162064

\section{FUNDING}

The authors received funding for this work through the National Cancer Institute (R21 CA186976), Brains Together for a Cure, The National Institute of Neurologic Disease and Stroke (R21 NS094765, R56 NS094765, and R01 NS103212), the Mayo ClinicKoch Institute Collaboration, the Mayo Clinic Graduate School of Biomedical Sciences, the Mayo Clinic College of Medicine, and the Mayo Foundation for Medical Education and Research.

12. Cumba Garcia LM, Huseby Kelcher AM, Malo CS, Johnson AJ. Superior isolation of antigen-specific brain infiltrating $\mathrm{T}$ cells using manual homogenization technique. J Immunol Methods (2016) 439:23-8. doi: 10.1016/j.jim.2016.09.002

13. McDole J, Johnson AJ, Pirko I. The role of $\mathrm{CD}^{+}{ }^{+}$T-cells in lesion formation and axonal dysfunction in multiple sclerosis. Neurol Res. (2006) 28:256-61. doi: $10.1179 / 016164106 \times 98125$

14. Voron T, Colussi O, Marcheteau E, Pernot S, Nizard M, Pointet AL, et al. VEGF-A modulates expression of inhibitory checkpoints on CD8 ${ }^{+}$ T cells in tumors. J Exp Med. (2015) 212:139-48. doi: 10.1084/jem.201 40559

15. Kumler I, Christiansen OG, Nielsen DL. A systematic review of bevacizumab efficacy in breast cancer. Cancer Treat Rev. (2014) 40:960-73. doi: 10.1016/j.ctrv.2014.05.006

16. Qu CY, Zheng Y, Zhou M, Zhang Y, Shen F, Cao J, et al. Value of bevacizumab in treatment of colorectal cancer: a meta-analysis. World J Gastroenterol. (2015) 21:5072-80. doi: 10.3748/wjg.v21.i1 6.5072

17. Ciombor KK, Wu C, Goldberg RM. Recent therapeutic advances in the treatment of colorectal cancer. Annu Rev Med. (2015) 66:83-95. doi: 10.1146/annurev-med-051513-102539

18. Ernst B, Anderson KS. Immunotherapy for the treatment of breast cancer. Curr Oncol Rep. (2015) 17:5. doi: 10.1007/s11912-014-0426-9

Conflict of Interest Statement: The authors declare that the research was conducted in the absence of any commercial or financial relationships that could be construed as a potential conflict of interest.

The handling Editor declared a shared affiliation, though no other collaboration, with the authors.

Copyright (c) 2018 Malo, Khadka, Ayasoufi, Jin, AbouChehade, Hansen, Iezzi, Pavelko and Johnson. This is an open-access article distributed under the terms of the Creative Commons Attribution License (CC BY). The use, distribution or reproduction in other forums is permitted, provided the original author(s) and the copyright owner(s) are credited and that the original publication in this journal is cited, in accordance with accepted academic practice. No use, distribution or reproduction is permitted which does not comply with these terms. 